\title{
The predictors of periodontal disease progression following treatment need more research
}

\author{
Where individuals have initial-cause-related periodontal therapy and after \\ initial therapy, are the residual probing depth, bleeding on probing and \\ furcation status predictors of further attachment and tooth loss?
}

\begin{abstract}
Renvert S, Persson GR. A systematic review on the use of residual probing depth, bleeding on probing and furcation status following initial periodontal therapy to predict further attachment and tooth loss. J Clin Periodontol 2002; 29(Suppl. 3):S82-S89
\end{abstract}

Data sources Sources used were MEDLINE, Embase and the Cochrane Oral Health Group Trials Register. Reference lists from relevant articles were hand-searched, as were selected journals. Only English-language studies were included.

Study selection Clinical trials and cohort studies were selected when they lasted a minimum of 12 months' after initial healing and addressed the predictive value of residual probing depths (PD), bleeding on probing and furcation involvement in determining further loss of attachment and tooth loss following initial-cause-related periodontal therapy (ICRT).

Data extraction and synthesis Information regarding quality and study characteristics was extracted independently by two reviewers. Kappa scores determined their agreement.

Results Only one study of 16 subjects provided patient-based data from longitudinal case studies related to ICRT and provided treatment outcomes with data from initial follow-up at 3 months through to 12 months after initial therapy.

Conclusions Data based on a single study suggest that residual probing depths may be predictive of further disease progression. There are clear implications for carefully designed multicentre randomised clinical control trials.

\section{Commentary}

One of the central tenets of clinical periodontics is that deep periodontal pockets, once established, enhance the extent or rate of attachment-loss in both untreated and treated cases. The issue is a critical one, given the implications of pocket elimination as a goal of periodontal therapy. The review's results, which indicate that residual PD are predictive of further disease progression, support this view. There are nevertheless significant caveats and a strict interpretation leads to other complications.

This rigorous systematic review examined 47 studies, but only one of these met all inclusion criteria and it is the only one upon which conclusions might be based. Further, even in this work only 16 subjects were followed over some 3 and a half years. One is left wondering, therefore, whether the continued loss of attachment was unique to this group of patients, or if it resulted from incomplete treatment, non-responsive individuals or recurrent disease.
It is notable that the systematic review evaluated residual PD, bleeding on probing and furcation involvement as prognostic indicators. In defining their inclusion and exclusion criteria the authors dealt with important considerations such as subjects versus sites as the unit of measure, chronic versus aggressive forms of disease, and variations in the interventions provided. Moreover, the authors' argument for choosing subjects rather than sites in their analysis is rational for biological and statistical reasons.

In the final analysis, unfortunately, the evidence remains equivocal. As William Lavine has pointed out, we may still be, "lost in the pocket". ${ }^{1}$ If we are inclined to accept the authors' conclusion here, that residual deep pockets are predictive of attachment-loss in treated cases, then we would be even more compelled to accept the conclusion that shallow sulci are predictive of attachment-loss in untreated cases. The latter conclusion is logically inescapable, albeit clinically useless, given that people are not born with periodontal disease and that periodontal disease initially develops in subjects with a healthy periodontium (ie, no pockets). ${ }^{2}$

So where does this leave us? The issue remains potentially divisive. On one hand, lack of evidence does not necessarily mean that the concept is wrong. On the other hand, the long-standing dearth of evidence does limit the likelihood of its validity. The review's authors correctly conclude that more controlled clinical studies are necessary to adequately address the question. Until then, clinicians should continue to treat their patients according to their professional judgement and experience.

\section{Practice point}

- Limited evidence suggests that residual probing depths may be predictive of further disease progression.

\section{Cataldo W Leone}

Department of Periodontology and Oral Biology, Boston University Goldman School of Dental Medicine, Boston, Massachusetts, USA

1. Lavine WS, Greenblatt IM. Lost in the pocket: the current status of periodontal diagnosis. Technol Health Care 1996; 4:317-322.

2. Tanner AC, Kent Jr RL, Maiden MF, Macuch PJ, Taubman MA. Serum IgG reactivity to subgingival bacteria in initial periodontitis, gingivitis and healthy subjects. J Clin Periodontol 2000; 27:473-480.

Evidence-Based Dentistry (2003) 4, 59.

doi:10.1038/sj.ebd.6400202 